\title{
Different levels of hyphal self-incompatibility modulate interconnectedness of mycorrhizal networks in three arbuscular mycorrhizal fungi within the Glomeraceae
}

\author{
Alessandra Pepe ${ }^{1}$, Manuela Giovannetti ${ }^{1}$, Cristiana Sbrana ${ }^{2}$ \\ 1. Department of Agriculture, Food and Environment University of Pisa Pisa Italy \\ 2. CNR, Institute of Agricultural Biology and Biotechnology UOS Pisa Pisa Italy
}

\begin{abstract}
Arbuscular mycorrhizal fungi (AMF) live in symbiosis with most plant species and produce underground extraradical hyphal networks functional in the uptake and translocation of mineral nutrients from the soil to host plants. This work investigated whether fungal genotype can affect patterns of interconnections and structural traits of extraradical mycelium (ERM), by comparing three Glomeraceae species growing in symbiosis with five plant hosts. An isolate of Funneliformis coronatus consistently showed low ability to form interconnected ERM and self-incompatibility that represented up to $21 \%$ of hyphal contacts. The frequency of post-fusion self-incompatible interactions, never detected before in AMF extraradical networks, was $8.9 \%$. In $F$. coronatus ERM, the percentage of hyphal contacts leading to perfect hyphal fusions was 1.2-7.7, while it ranged from 25.8-48 to 35.6-53.6 in Rhizophagus intraradices and Funneliformis mosseae, respectively. Low interconnectedness of $F$. coronatus ERM resulted also from a very high number of non-interacting contacts $(83.2 \%)$. Such findings show that AMF genotypes in Glomeraceae can differ significantly in anastomosis behaviour and that ERM interconnectedness is modulated by the fungal symbiont, as $F$. coronatus consistently formed poorly interconnected networks when growing in symbiosis with five different host plants and in the asymbiotic stage. Structural traits, such as extent, density and hyphal selfcompatibility/incompatibility, may represent key factors for the differential performance of AMF, by affecting fungal absorbing surface and foraging ability and thus nutrient flow from soil to host roots.
\end{abstract}

Keywords

Arbuscular mycorrhizal fungi, Extraradical networks, Hyphal anastomosis, Mycelial interconnectedness, Hyphal incompatibility, Funneliformis coronatus

\section{Introduction}

Arbuscular mycorrhizal (AM) fungi (AMF), which belong to the Phylum Glomeromycota, are obligate biotrophs obtaining sugars from their host plants, in exchange of mineral nutrients absorbed from the soil and translocated by means of extraradical hyphal networks spreading from colonised roots into the surrounding soil (Giovannetti et al. 2015). Such networks may extend from 2.7 to $20.5 \mathrm{~m}$ per gram of soil, depending on the fungal species (Giovannetti and Avio 2002; Mikkelsen et al. 2008). The ability to produce large and interconnected extraradical mycelium (ERM) increases the fungal absorbing surface and foraging ability and facilitates the translocation and flow of mineral nutrients from soil to host plants, affecting plant growth and nutrition (Avio et al. 2006). Moreover, a high level of interconnectedness between contiguous mycorrhizal networks creates indefinitely large numbers of linkages through which nutrients can flow between different neighbouring plants (Giovannetti et al. 2004; Mikkelsen et al. 2008).

A non-destructive approach using root observation chambers provides qualitative information on the architecture and development of AMF extraradical networks (Friese and Allen 1991), while an in vivo bidimensional experimental system allows the visualisation and quantification of intact ERM whose lengths can range from $5169 \mathrm{~mm}$ to $7471 \mathrm{~mm}$ for Funneliformis mosseae in association with Thymus vulgaris and Allium porrum, respectively, with a mean growth rate of 738-1067 mm day-1, depending on the host plant (Giovannetti et al. 2001). Lower values have been detected in a tri-dimensional soil system, from 3.1 to 
$3.8 \mathrm{~mm}$ day -1 in $F$. mosseae and Funneliformis caledonius ERM spreading from Trifolium subterraneum mycorrhizal roots (Mikkelsen et al. 2008).

AMF belonging to the Glomeraceae form fusions (anastomoses) between contacting hyphae, while members of the Gigasporaceae, Ambisporaceae and Paraglomeraceae do not show evidence of being able to fuse in vivo (Purin and Morton 2011), in accordance with previous data on the inability of asymbiotic mycelium of AMF belonging to Gigasporaceae to produce anastomoses (Giovannetti et al. 1999; 2003). Different isolates of Gigasporaceae also show distinct patterns of anastomosis in root-organ cultures (ROC), where about $95 \%$ of fusions can occur within the same hypha, by a mechanism known as wound healing (de la Providencia et al. 2005; Voets et al. 2006).

Data available on in vivo anastomosis formation in Glomeraceae ERM are limited to isolates of $F$. mosseae and Rhizophagus spp., where frequencies range from 44 to $64 \%$ and from 2.4 to $66.6 \%$, respectively (Giovannetti et al. 1999; Avio et al. 2006; Purin and Morton 2011, 2013). These studies have provided evidence for the occurrence of self-incompatible interactions preceding hyphal fusions and consisting in cross-wall formation, protoplasm retraction and death of the respective hyphal compartment. The frequencies of such prefusion self-incompatibility have been reported to range from 5 to $32.1 \%$ of contacts, while no data on postfusion incompatible reactions are available (Purin and Morton 2011; Sbrana et al. 2011; Purin and Morton 2013).

In this work, an in vivo system was used to analyse the occurrence and frequency of anastomoses and of incompatible interactions, both preceding and following hyphal fusions, in ERM formed by isolates of three AMF within the Glomeraceae- $F$. mosseae, Rhizophagus intraradices and Funneliformis coronatusspreading from the roots of three different host plants, Lactuca sativa, Cichorium intybus (three varieties) and Valerianella locusta. The comparison between the AMF isolates showed significant differences among fungal genotypes in ERM self-incompatibility, which affected the structure of networks and the pattern of hyphal interconnectedness.

\section{Materials and methods}

Fungal material

Isolates IMA1, IMA6 and IMA3 of $F$. mosseae (T.H. Nicolson \& Gerd.) C. Walker \& A. Schüßler, $R$. intraradices (N.C. Schenck \& G.S. Sm.) C. Walker \& A. Schüßler and F. coronatus (Giovann.) C. Walker \& A. Schüßler, respectively, were maintained in Medicago sativa L. pot cultures in the Microbiology Laboratory Collection of the Department of Agriculture, Food and Environment, University of Pisa, Italy. AMF inoculum was obtained from the pot culture soil after wet sieving through a $100-\mu \mathrm{m}$-mesh size sieve, and spores or sporocarps, mycelium and colonised roots in the retained fraction were added to test plants (see below).

Plant material

AMF plant hosts were as follows: L. sativa L. var. crispa, curly green leaf lettuce (LSC), C. intybus var. foliosum, sugar chicory of Trieste (CIF-T), C. intybus var. foliosum, cutting chicory Spadona (CIF-S), $C$. intybus var. foliosum, golden-leaved Trieste chicory (CIF-B) and V. locusta L. (corn salad, VL). Surfacesterilised seeds were germinated in moist sterile grit. After 10 days, the root system of each seedling was AMF inoculated, in 6-cm-diameter pots containing sterile calcined attapulgite clay (Oil Dri, Chicago, IL). At least five replicate plants for each fungal and host species were prepared. Pots were closed in Sun-Transparent Bags (Sigma Aldrich s.r.l. Milan, Italy) and maintained in a growth chamber with $25{ }^{\circ} \mathrm{C}$ day and $21{ }^{\circ} \mathrm{C}$ night temperature, 16:8-h light/dark cycle.

\section{Experimental design}

Plants were harvested after 4 weeks. Roots were gently removed from the substrate by immersion in water and checked for the occurrence of extraradical hyphae, spores and sporocarps. The spores and sporocarps were 
carefully removed with forceps under a Leica M 205C dissecting microscope (Leica, Milano, Italy). Mycorrhizal colonisation was confirmed by clearing and staining of sample plant roots, using lactic acid instead of lactophenol (Phillips and Hayman 1970). The roots of each plant were placed between two semicircular 13-cm-diameter Millipore ${ }^{\mathrm{TM}}$ membranes (root sandwiches) and then transferred into 14-cmdiameter Petri dishes containing sterile quartz grit and maintained in the growth chamber (Online Resource 1). During incubation, plants were fertilised with modified Long Ashton nutrient solution (with half-strength $\mathrm{P}$ concentration). The root sandwiches were opened after 1 month, and roots and ERM growing on the membranes were stained for succinate dehydrogenase (SDH) activity (Online Resource 1) (Smith and Gianinazzi-Pearson 1990). Deposition of formazan salts in intraradical hyphae allowed the measurement of viable colonised root length using the gridline intersect method (Giovannetti and Mosse 1980) under the Leica dissecting microscope. After the SDH test, the same membranes were stained with Trypan blue in lactic acid $(0.05 \%)$ to assess hyphal density, under the dissecting microscope.

Mean hyphal density within ERM (hyphal length $\mathrm{mm}-2$ ) was assessed with the gridline intersect method by estimating the length of hyphae in five areas of $16 \mathrm{~mm} 2$ for each membrane, on three replicate membranes, using a grid eyepiece. On the same membranes, the area explored by ERM was determined using a transparent 5-mm-square grid. Total hyphal length was calculated from measured data, multiplying mean hyphal density by the area explored by the mycelial network.

Areas of membranes covered by ERM were cut, mounted on microscope slides and examined under a ReichertJung (Vienna, Austria) Polyvar microscope. At least three samples from each replicate membrane were analysed. Hyphal contacts were counted at magnifications of $\times 125-500$ and classified into four categories at a magnification of $\times 1250$, on the basis of hyphal viability and protoplasmic continuity established between fusing hyphae, evidenced by the deposition of formazan salts. Perfect fusions were recorded when hyphae anastomosed and protoplasm continuity could be verified by SDH staining. Pre-fusion incompatibility was scored when protoplasm retraction and septa were observed before fusion in hyphae showing morphological changes indicative of the first step of anastomosis formation (hyphal tip swelling, hyphal homing). Post-fusion incompatibility was scored when protoplasm withdrawal occurred in one or both hyphae, and septa isolating fused hyphal compartments were formed after anastomosis. Hyphal contacts were scored as non-interacting when hyphae crossed with no interactions. Overall, the total number of hyphal contacts analysed ranged from 3325 to 3543 for $F$. mosseae and $R$. intraradices and 5522 for $F$. coronatus. Frequencies in each category of hyphal contact were calculated as a percentage of total hyphal contacts detected.

To visualise the occurrence and location of nuclei in contacting hyphae, some membranes were stained with $5 \mu \mathrm{g} \mathrm{ml}-1$ diamidinophenylindole (DAPI) in a $1: 1(\mathrm{v} / \mathrm{v})$ water/glycerol solution and observed under epifluorescence with the Polyvar microscope using the filter combination U1 (BP 330-380, LP 418, DS 420). To detect incompatibility reactions in hyphal contacts, the presence of wall thickenings and retraction septa was investigated on some membranes, which were mounted in a $0.01 \%(\mathrm{w} / \mathrm{v})$ solution of Calcofluor White (Sigma-Aldrich s.r.l. Milan, Italy) and observed under epifluorescence with the filter combination U1. The microscope was equipped with a Leica DFC 450C video camera (Leica, Milano, Italy) connected to a personal computer, and pictures were captured using Leica Application Suite software (version 4.0.0).

To compare anastomosis ability of ERM vs. asymbiotic mycelium of $F$. coronatus, spores were rinsed in distilled water and placed in two rows of five, approximately $0.5 \mathrm{~cm}$ apart, on cellulose ester membranes (Millipore, $47-\mathrm{mm}$ diameter; $0.45-\mu \mathrm{m}$ pore size, 20 replicates). Membranes were closed, incubated, stained and observed as described in Giovannetti et al. (2003).

\section{Data analysis}

Jeffreys $95 \%$ confidence limits (95\% CL) on hyphal interaction frequencies were calculated using EpiTools epidemiological calculators (http://epitools.ausvet.com.au). Percentage data were subjected to arcsine transformation before carrying out statistical analyses. A two-way ANOVA was performed to assess the significance of the two factors involved (fungal species and host plant species) on host root colonisation and 
each category of hyphal interaction. A one-way ANOVA was used to detect significant differences between data obtained for different plant species or different fungal species within each category of hyphal interaction.

Results

Interconnectedness of mycorrhizal networks

The interconnected ERM spreading from plant root systems was visible after SDH and Trypan blue staining, which allowed the detection of successful hyphal fusions, characterised by protoplasm continuity and formazan salt depositions (Fig. 1). The isolate IMA3 of $F$. coronatus showed a very low ability to form perfect anastomoses and interconnected networks (Fig. 1), with frequencies ranging from $1.2 \%$ (95\% CL 0.6-2.2\%) to $7.7 \%$ (95\% CL 5.8-10.0\%) (Fig. 2). In contrast, isolates of F. mosseae and R. intraradices showed average values of anastomosis frequency ranging from 25.8 (95\% CL 22.0-29.8 \%) to $48.0 \%$ (95\% CL 44.0-52.0\%) and from $35.6 \%$ (95 \% CL 31.7-39.7\%) to 53.6 (95\% CL 49.0-58.2 \%), respectively (Fig. 2). Two-way ANOVA showed that fungal genotype significantly affected network interconnectedness and that AMF $\times$ plant interaction was not significant (Table 1). Two-way and one-way ANOVA post hoc tests evidenced significantly different interconnectedness values for networks produced by each AMF genotype, independent of host plant species and variety tested $(\mathrm{P}=0.005)$. 


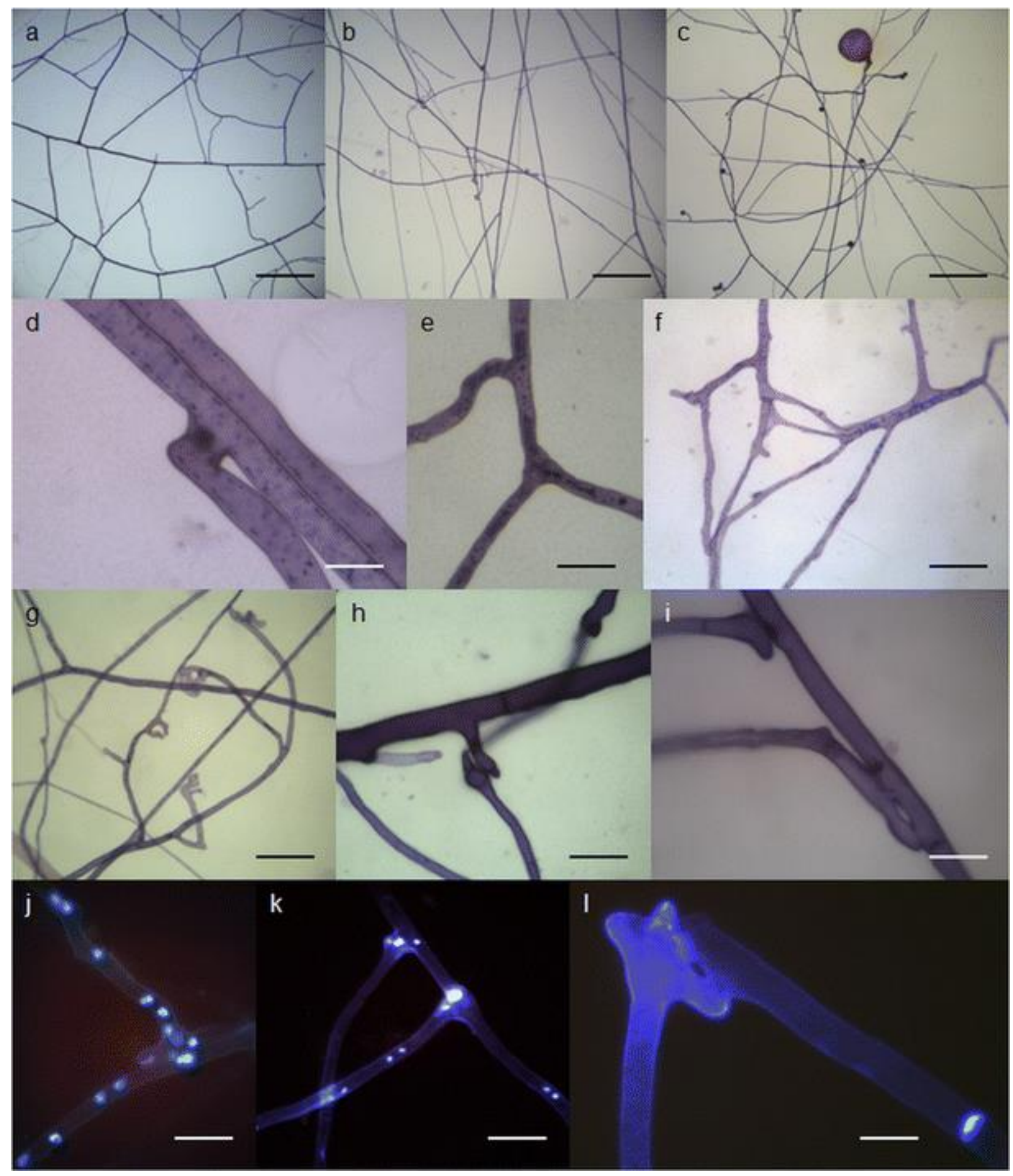

Fig. 1. Light and epifluorescence micrographs showing differential features of mycelium produced by Funneliformis mosseae, Funneliformis coronatus and Rhizophagus intraradices. a Highly interconnected extraradical network developed by $F$. mosseae in symbiosis with Cichorium intybus. Scale bar $=60 \mu \mathrm{m}$. b Low interconnected extraradical network produced by $F$. coronatus in symbiosis with $C$. intybus. Scale bar $=60 \mu \mathrm{m}$. c Asymbiotic mycelium produced by a $F$. coronatus germinated spore showing many non-interacting hyphal contacts. Scale bar $=60 \mu \mathrm{m}$. d Protoplasm continuity in a perfect fusion in the extraradical network formed by $R$. intraradices after localization of succinate dehydrogenase activity (SDH) and Trypan blue staining. Scale $\mathrm{bar}=9 \mu \mathrm{m}$. e Protoplasm continuity in perfect fusions in the extraradical network formed by $F$. coronatus after localization of SDH and Trypan blue staining. Scale bar $=20 \mu \mathrm{m}$. f, g Pre-fusion and no hyphal interactions occurring within the extraradical network produced by $F$. coronatus in symbiosis with $C$. intybus. Scale bars $=30 \mu \mathrm{m}$. h Pre-fusion incompatible interaction in the extraradical network of $F$. coronatus, characterised by empty and septated approaching hyphae. Scale bar $=20 \mu \mathrm{m}$. i Post-fusion incompatible interactions in the 
extraradical network formed by $F$. coronatus, showing formation of septa within fused hyphae. Scale bar $=25 \mu \mathrm{m}$. j, k DAPI staining showing the occurrence of nuclei in $F$. mosseae (j) and $F$. coronatus $(\mathrm{k})$ anastomosing hyphae within the extraradical network. Scale bars $=20 \mu \mathrm{m} .1$ Calcofluor staining of a postfusion incompatible interaction between hyphae of the extraradical network produced by $F$. coronatus, revealing wall thickening at the fusion site and the formation of a cross-wall. Scale bar $=8 \mu \mathrm{m}$

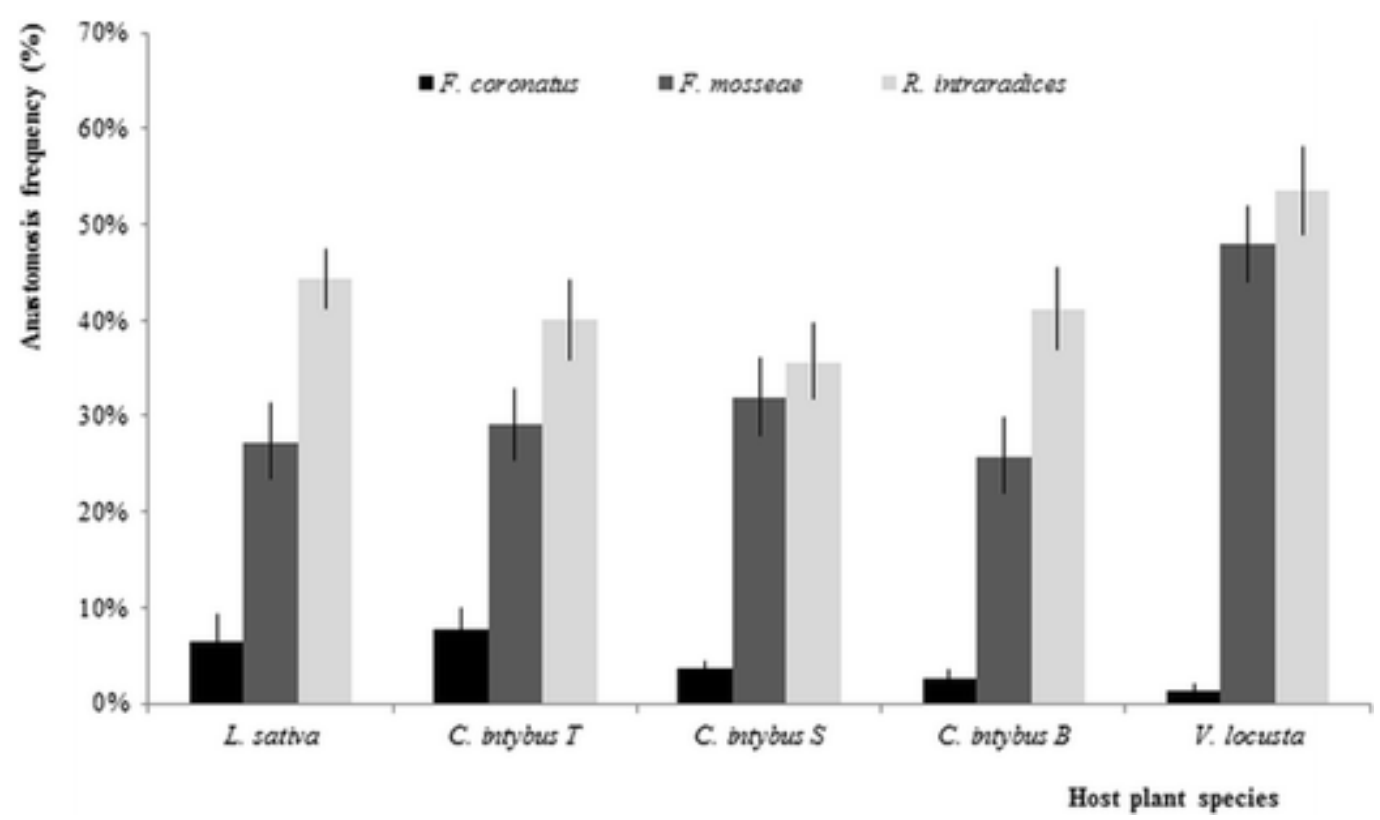

Fig. 2. Mean frequencies (95\% confidence limits) of hyphal anastomosis detected within extraradical mycorrhizal networks formed by Funneliformis mosseae, Funneliformis coronatus and Rhizophagus intraradices with different plant species. LSC Lactuca sativa, curly green leaf lettuce; CIF-T Cichorium intybus, sugar chicory of Trieste; CIF-S Cichorium intybus, cutting chicory Spadona; CIF-B Cichorium intybus, broad-leaved cutting chicory; VL Valerianella locusta

Table 1. $\mathrm{P}$ values of main effects of factors (fungal species and host plant) and interactions in the analysis of structural parameters and interconnectedness of extraradical networks produced by isolates of Funneliformis mosseae, Funneliformis coronatus and Rhizophagus intraradices in symbiosis with Lactuca sativa, Cichorium intybus and Valerianella locusta

Hyphal density Explored area Hyphal length Anastomosis frequency

\begin{tabular}{lcccc}
\hline AMF species & 0.007 & 0.004 & 0.004 & 0.000 \\
Host plant & 0.004 & 0.348 & 0.002 & 0.092 \\
AMF × plant & 0.000 & 0.307 & 0.025 & 0.065 \\
\hline
\end{tabular}


Observation of DAPI-stained membrane areas showed the occurrence of nuclei in fusion bridges in ERM networks produced by the three AMF species analysed, even in the rare perfect anastomoses occurring in $F$. coronatus (Fig. 1).

Incompatible interactions in $F$. coronatus mycorrhizal networks

Mycorrhizal networks formed by $F$. coronatus exhibited a low rate of perfect anastomosis and a very high percentage of contacts producing no hyphal interactions (71.6-93.6\% of contacts) (Fig. 3). Moreover, preand post-fusion incompatible hyphal interactions occurred at variable rates, depending on host plant. Incompatible interactions were characterised by protoplasm retraction followed by septa formation (crosswalls), isolating the contacting hypha or the compartment formed after hyphal fusion (Fig. 1). ERM produced in symbiosis with $C$. intybus CIF-T showed the highest percentages of incompatible fusions $(17.3 \%, 95 \% \mathrm{CL}$ 14.5-20.3) whereas that formed in symbiosis with V. locusta had the lowest values (4.3\%, $95 \%$ CL 3.0-5.8) (Fig. 3). Pre-fusion incompatibility, where retraction of protoplasm followed by septation occurred in one or both contacting hyphae (Fig. 1), showed similar values for F. coronatus ERM produced with all host plants except V. locusta. On the contrary, incompatible interactions occurring after hyphal fusion showed variable values between ERMs produced with the different host species (Fig. 3). Results of ANOVA confirmed significant differences $(\mathrm{P}<0.001)$ between all the plants used as AMF hosts when assessing $F$. coronatus ERM rates of hyphal contacts leading to perfect fusions, no interactions or post-fusion incompatibility, whereas prefusion incompatible interaction percentages were significantly lower only in ERM produced with V. locusta $(\mathrm{P}<0.001)$.

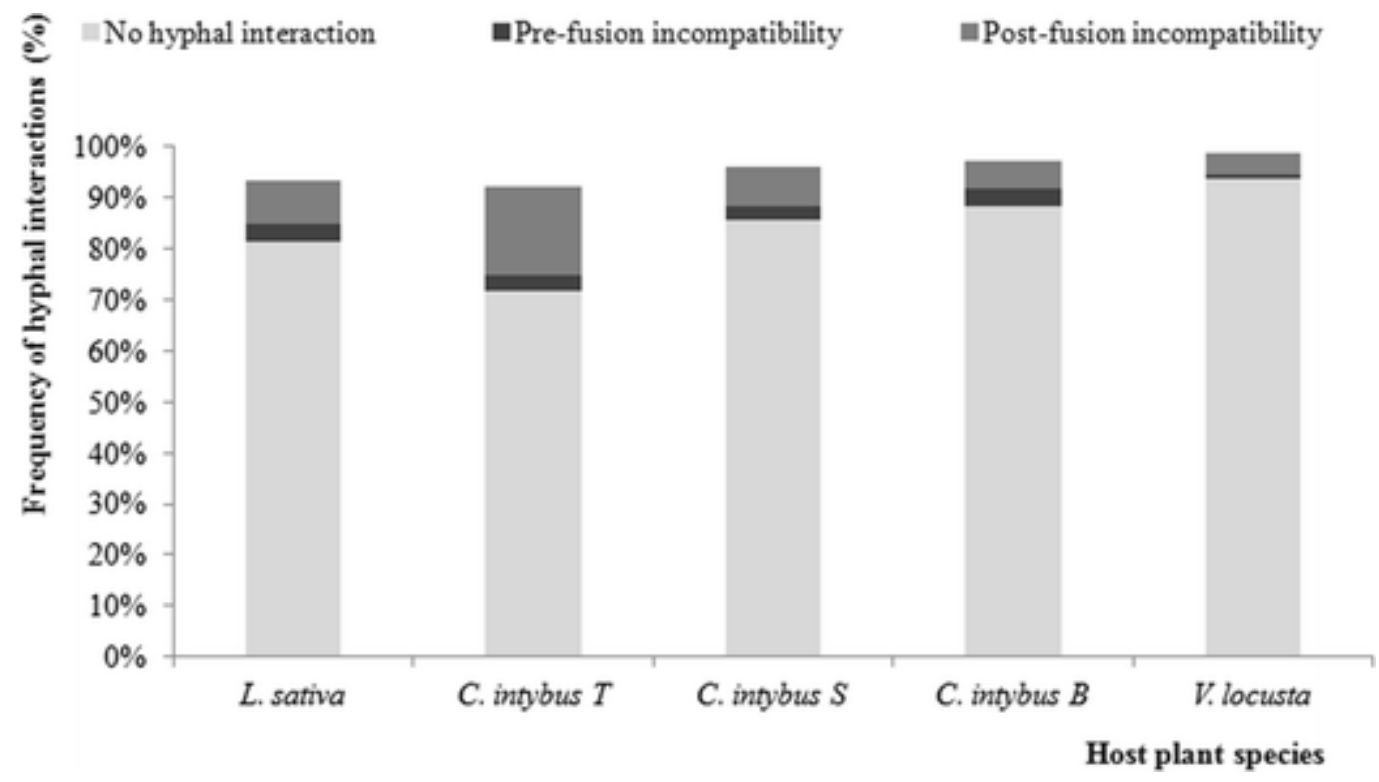

Fig. 3. Mean frequencies (95\% confidence limits) of hyphal interactions recorded within extraradical mycorrhizal networks formed by Funneliformis coronatus with different plant species. LSC Lactuca sativa, curly green leaf lettuce; CIF-T Cichorium intybus, sugar chicory of Trieste; CIF-S Cichorium intybus, cutting chicory Spadona; CIF-B Cichorium intybus, broad-leaved cutting chicory; VL Valerianella locusta

Comparison of data obtained from $F$. coronatus ERM analysis with those recorded on asymbiotic mycelium produced by germinating spores of the same fungus confirmed a low ability of this AMF to anastomose (Fig. 1), although some differences were evident between the two developmental stages (Table 2). Asymbiotic mycelium showed a high frequency of hyphal contacts without interactions $(91.2 \%)$ and low frequencies of perfect fusions, incompatible fusions and pre-fusion incompatible interactions (Table 2). Statistical analyses of data obtained for asymbiotic mycelium and for ERM showed a significantly lower frequency of incompatible fusions, paralleled by a significantly higher frequency of non-interacting contacts $(\mathrm{F}=19.512$, 
$\mathrm{P}<0.001$ and $\mathrm{F}=6.897, \mathrm{P}=0.014$, respectively) in the asymbiotic stage, whereas no significant differences were detected in perfect fusion rates $(\mathrm{F}=0.842, \mathrm{P}=0.367)$ and in pre-fusion incompatible interaction frequencies $(\mathrm{F}=2.368, \mathrm{P}=0.136)$ between the two developmental stages.

Table 2. Mean frequencies (95\% confidence limits) of Funneliformis coronatus hyphal interactions detected either within extraradical networks formed with different plant species (symbiotic stage) or within mycelium originating from germinated spores (asymbiotic stage)

\begin{tabular}{lcc} 
& Symbiotic stage & Asymbiotic stage \\
\hline Perfect fusions (\%) & $4.4(3.9-4.9)$ & $4.1(3.1-5.4)$ \\
No hyphal interactions (\%) & $83.2(82.3-84.1)$ & $91.2(89.4-92.7)$ \\
Pre-fusion incompatibility (\%) & $2.7(2.3-3.1)$ & $1.5(0.9-2.4)$ \\
Post-fusion incompatibility (\%) & $8.9(8.2-9.6)$ & $3.2(2.3-4.3)$ \\
\hline
\end{tabular}

Table 3. Mean frequencies ( \pm standard error of means) of mycorrhizal colonization by Funneliformis mosseae, Funneliformis coronatus and Rhizophagus intraradices of different plant species

Mycorrhizal colonization (\%)

F. mosseae $\quad F$. coronatus $\quad R$. intraradices

\begin{tabular}{lrrr}
\hline LSC8 & $63.9 \pm 4.9 \mathrm{a}$ & $57.7 \pm 0.2 \mathrm{a}$ & $64.8 \pm 2.7 \mathrm{a}$ \\
CIF-T & $67.6 \pm 1.4 \mathrm{a}$ & $58.2 \pm 6.3 \mathrm{ab}$ & $44.0 \pm 1.6 \mathrm{~b}$ \\
CIF-S & $63.6 \pm 0.1 \mathrm{a}$ & $48.1 \pm 4.3 \mathrm{ab}$ & $54.2 \pm 4.1 \mathrm{ab}$ \\
& & & \\
CIF-B & $37.6 \pm 1.6 \mathrm{~b}$ & $39.6 \pm 3.1 \mathrm{~b}$ & $43.5 \pm 2.9 \mathrm{~b}$ \\
& & & \\
VL & $59.7 \pm 2.5 \mathrm{a}$ & $59.5 \pm 0.5 \mathrm{ab}$ & $52.1 \pm 1.3 \mathrm{~b}$
\end{tabular}

In columns, means followed by different letters are significantly different for $\mathrm{P}=0.01$. LSC Lactuca sativa, curly green leaf lettuce; CIF-T Cichorium intybus, sugar chicory of Trieste; CIF-S Cichorium intybus, cutting chicory Spadona; CIF-B Cichorium intybus, broad-leaved cutting chicory; VL Valerianella locusta 
Mycorrhizal colonisation

Colonised root lengths recorded in AMF-inoculated L. sativa, C. intybus and V. locusta ranged between 38 and $68 \%$, depending on the host-fungus combination. The three fungal species produced extensive root colonisation in all plants, except for $C$. intybus CIF-B, which showed the lowest colonisation levels with all the AMF tested (Table 3).

Hyphal density and length of mycorrhizal networks

The bidimensional model system allowed the detection of marked differences among structural traits of ERM formed by $F$. mosseae, $F$. coronatus and $R$. intraradices. Specifically, $F$. coronatus colonising V. locusta showed the lowest values of total mycelial length $(9.7 \pm 1.1 \mathrm{~m})$, while the highest values were recorded in $F$. mosseae colonising $C$. intybus CIF-T (64.7 $\pm 5.7 \mathrm{~m})$ (Table 4).

Table 4. Mean values ( \pm standard errors of the mean) of structural parameters of extraradical networks formed by Funneliformis mosseae, Funneliformis coronatus and Rhizophagus intraradices with different plant species

Hyphal density (mm/mm-2) Explored area (mm2) Hyphal length (m)

\begin{tabular}{|c|c|c|c|c|}
\hline \multirow[t]{5}{*}{ F. mosseae } & LSC & $4.7 \pm 0.2 \mathrm{a}$ & $5516.7 \pm 247.6$ & $26.1 \pm 1.9 \mathrm{a}$ \\
\hline & CIF-T & $9.8 \pm 0.5 b$ & $6566.7 \pm 272.5$ & $64.7 \pm 5.7 \mathrm{~b}$ \\
\hline & CIF-S & $5.4 \pm 1.1 \mathrm{a}$ & $5783.3 \pm 1567.9$ & $28.6 \pm 3.1 \mathrm{a}$ \\
\hline & CIF-B & $9.4 \pm 1.1 \mathrm{~b}$ & $5483.3 \pm 677.4$ & $52.0 \pm 9.7 \mathrm{ab}$ \\
\hline & VL & $8.3 \pm 0.6 \mathrm{ab}$ & $5887.5 \pm 812.5$ & $47.8 \pm 11.2 \mathrm{ab}$ \\
\hline \multirow[t]{5}{*}{ F. coronatus } & LSC & $5.5 \pm 1.2 \mathrm{ab}$ & $3887.5 \pm 762.5$ & $22.6 \pm 10.1 \mathrm{a}$ \\
\hline & CIF-T & $7.6 \pm 1.2 \mathrm{bc}$ & $4883.3 \pm 685.3$ & $38.1 \pm 10.1 \mathrm{a}$ \\
\hline & CIF-S & $9.6 \pm 0.9 \mathrm{c}$ & $4241.7 \pm 1061.9$ & $41.6 \pm 12.1 \mathrm{a}$ \\
\hline & CIF-B & $5.4 \pm 0.6 \mathrm{ab}$ & $4933.3 \pm 563.9$ & $26.2 \pm 1.0 \mathrm{a}$ \\
\hline & VL & $3.7 \pm 0.2 \mathrm{a}$ & $2591.7 \pm 180.5$ & $9.7 \pm 1.1 \mathrm{a}$ \\
\hline \multirow[t]{3}{*}{ R. intraradices } & LSC & $9.1 \pm 0.9 \mathrm{~b}$ & $4725.0 \pm 337.6$ & $43.1 \pm 5.8 \mathrm{ab}$ \\
\hline & CIF-T & $9.9 \pm 0.2 \mathrm{~b}$ & $6358.3 \pm 158.3$ & $62.8 \pm 1.3 \mathrm{~b}$ \\
\hline & CIF-S & $5.5 \pm 0.5 \mathrm{a}$ & $4416.7 \pm 903.0$ & $24.6 \pm 5.7 \mathrm{a}$ \\
\hline
\end{tabular}


Hyphal density (mm/mm-2) Explored area (mm2) Hyphal length (m)

\begin{tabular}{llrl}
\hline CIF-B & $8.2 \pm 0.2 \mathrm{~b}$ & $5616.7 \pm 433.1$ & $46.0 \pm 2.5 \mathrm{ab}$ \\
VL & $7.7 \pm 0.2 \mathrm{ab}$ & $6058.3 \pm 939.0$ & $46.2 \pm 6.4 \mathrm{ab}$ \\
\hline
\end{tabular}

In columns, means followed by different letters are significantly different for $\mathrm{P}=0.01$. LSC Lactuca sativa, curly green leaf lettuce; CIF-T Cichorium intybus, sugar chicory of Trieste; CIF-S Cichorium intybus, cutting chicory Spadona; CIF-B Cichorium intybus, broad-leaved cutting chicory; VL Valerianella locusta

A large variation in values of ERM hyphal density, ranging from $3.7 \pm 0.2$ to $9.9 \pm 0.2 \mathrm{~mm} \mathrm{~mm}-2$, was detected among plant-symbiont combinations. $F$. coronatus in symbiosis with V. locusta produced ERM with a significantly lower density, compared with that found in ERM developed with $C$. intybus CIF-S (Table 4). Networks formed by $F$. mosseae showed significantly higher densities when colonising two of the $C$. intybus varieties (CIF-T and CIF-B) compared to the other host plants, whereas $R$. intraradices hyphal density was significantly lower in networks formed with $C$. intybus CIF-S with respect to those formed with $C$. intybus CIF-T, CIF-B and L. sativa.

Results of two-way ANOVA showed that both fungal and plant identity significantly affected hyphal density and mycelial length and that $\mathrm{AMF} \times$ plant interaction was significant (Table 1). Post hoc tests evidenced that ERM hyphal density and length formed by symbionts with $C$. intybus CIF-T were significantly different compared with the other host plants, independent of fungal identity, and that $F$. coronatus ERM showed significantly lower density and length between the different host plants $(\mathrm{P}<0.001)$.

The explored area showed values ranging from $2591.7 \pm 180.5 \mathrm{~mm} 2$ in $F$. coronatus in symbiosis with V. locusta to $6566.7 \pm 272.5 \mathrm{~mm} 2$ in $F$. mosseae colonising $C$. intybus CIF-T (Table 4). Comparing explored area to total available surface $(9750 \mathrm{~mm} 2)$ among host-symbiont combinations, a high coverage of ERM was produced by all three AMF in symbiosis with $C$. intybus CIF-T $(67.3 \pm 2.8,50.1 \pm 7.0$ and $65.2 \pm 1.6 \%$ for $F$. mosseae, $F$. coronatus and $R$. intraradices, respectively). Results of two-way ANOVA showed that fungal genotype significantly affected the area explored by the mycorrhizal network and that AMF $\times$ plant interaction was not significant (Table 1). Post hoc tests confirmed significantly different values of the ERM explored area for $F$. coronatus across the different hosts $(\mathrm{P}<0.001)$.

\section{Discussion}

The present data indicate the inability of an $F$. coronatus isolate to produce highly interconnected extraradical networks, as a result of hyphal self-incompatibility and of a high frequency of non-interacting contacts. Such findings are contrary to what has been observed for other Glomeraceae and suggest that ERM interconnectedness is determined at the level of the fungal genotype, since $F$. coronatus consistently produced poorly interconnected networks when growing in symbiosis with five different host plants.

In $F$. coronatus ERM, the average frequency of post-fusion self-incompatibility, which has never been detected before in AMF within the Glomeraceae, was $8.9 \%$ of hyphal contacts, while pre-fusion incompatible interactions were detected in 0.86-3.5\% of contacts, consistent with previous data (Purin and Morton 2011, 2013; Sbrana et al. 2011). Genes mediating incompatibility are as yet unknown in AMF, although hyphal morphogenetic changes detected in incompatible interactions resemble those observed in other fungi, where divergence levels in the multiple het alleles shared by fusing hyphae trigger programmed cell death reactions (Glass and Kaneko 2003). The low ability of $F$. coronatus ERM to form perfect anastomoses was comparable to that of its asymbiotic mycelium, in contrast with the differential behaviour observed in the two developmental stages in Rhizophagus clarus isolates (Purin and Morton 2013). Further studies are needed to 
investigate whether the low anastomosing ability observed for extraradical and asymbiotic networks of the $F$. coronatus isolate IMA 3 is shared among other isolates belonging to the same species. No previous characterisation of ERM structural traits of putative functional significance has been performed on $F$. coronatus, which is currently distinguished from $F$. mosseae by spore subcellular structure (http://invam.wvu.edu/the-fungi/classification/glomaceae/funneliformis/coronatum) and phylogenetic analyses of SSU-ITS-LSU rDNA (Krüger et al. 2012). Data on the frequency of perfect anastomoses in $F$. mosseae and $R$. intraradices ERM are consistent with those obtained by other authors. In vivo ERM anastomosis frequencies in the Glomeraceae have been reported to vary among species and isolates, ranging from 2.4 to $67 \%$ of hyphal contacts (Purin and Morton 2013; Giovannetti et al. 2004; Avio et al. 2006). Such variability in AMF anastomosis behaviour suggests the involvement of multiple gene (or multiple allele) variation in determining the degree of vegetative compatibility in this group of fungi.

DAPI staining allowed the visualisation of nuclei in fusion bridges of ERM produced by the three AMF species analysed here, even in the rare perfect anastomoses occurring in $F$. coronatus, confirming previous observations (Croll et al. 2009). The migration of nuclei in hyphal bridges may be functional to information flow in ERM, possibly balancing the effects of segregation and genetic drift on fungal phenotypic characteristics and AMF-plant relationships (Angelard and Sanders 2011).

The present work also showed significant differences in structure and interconnectedness of ERM produced by the three AMF isolates among host plants, confirming that both fungal symbiont and plant host can affect network traits. Significant differences have been found in the interconnectedness of ERM produced by geographically and functionally different isolates of $F$. mosseae and $R$. intraradices in symbiosis with M. sativa (Avio et al. 2006), suggesting that physiological variability among co-specific isolates may entail systems controlling nutrient flow in different ERM regions, modulating hyphal growth, secondary root colonisation and spore production. Variability of ERM traits depending on the host plant has also been observed in vitro, where greater ERM growth was associated with chicory roots, compared with Medicago and carrot roots (Engelmoer and Kiers 2015), and in vivo, where ERM density and length were higher with cotton, cucumber, wheat and alfalfa, compared to other host plants (Ravnskov and Jakobsen 1995; Giovannetti et al. 2004; Smith et al. 2004).

Interconnectedness, density and length of ERM may affect fungal interactions with the environment and fungal-plant nutritional relationships. Accordingly, highly anastomosing AMF may be more capable of tolerating soil disturbance and grazing by the production of large and fast-growing networks (Helgason et al. 1998; Simard and Durrell 2004; Avio et al. 2013), whereas low interconnectedness or self-incompatibility may negatively affect AMF adaptation ability and fitness and reduce the development of common mycorrhizal networks (Engelmoer and Kiers 2015; Giovannetti et al. 2015). Network traits may also be of functional significance for nutrient flow within ERM, influencing symbiotic efficiency and plant growth, since ERM extent and interconnectedness can be positively correlated with host growth response variables and $\mathrm{P}$ content (Avio et al. 2006). An increasing number of genes encoding proteins for transport of mineral nutrients have been detected in ERM, and their expression has been monitored in response to the availability of mineral nutrients in ROCs (Gonzalez-Guerrero et al. 2005; Lopez-Pedrosa et al. 2006; Cappellazzo et al. 2008; Fiorilli et al. 2013). Further investigations need to focus on ERM functional significance, in order to reveal possible relationships between structural traits-such as extent, density and hyphal selfcompatibility/incompatibility —, the expression of mineral transporters and the ability to translocate nutrients to host plants, key factors for the differential performance of AMF.

Notes

Acknowledgments

This work represents part of A.P.'s PhD thesis project, which was funded by the University of Pisa. The financial support of the University of Pisa (Fondi di Ateneo) and of CNR is gratefully acknowledged. 


\section{References}

Angelard C, Sanders IR (2011) Effect of segregation and genetic exchange on arbuscular mycorrhizal fungi in colonization of roots. New Phytol 189:652-657

Avio L, Pellegrino E, Bonari E, Giovannetti M (2006) Functional diversity of arbuscular mycorrhizal fungal isolates in relation to extraradical mycelial networks. New Phytol 172:347-357

Avio L, Castaldini M, Fabiani A, Bedini S, Sbrana C, Turrini A et al (2013) Impact of nitrogen fertilization and soil tillage on arbuscular mycorrhizal fungal communities in a Mediterranean agroecosystem. Soil Biol Biochem 67:285-94

Cappellazzo G, Lanfranco L, Fitz M, Wipf D, Bonfante P (2008) Characterization of an amino acid permease from the endomycorrhizal fungus Glomus mosseae. Plant Physiol 147:429-437

Croll D, Giovannetti M, Koch AM, Sbrana C, Ehinger M, Lammers PJ, Sanders IR (2009) Nonself vegetative fusion and genetic exchange in the arbuscular mycorrhizal fungus Glomus intraradices. New Phytol 181:924937

de la Providencia IE, de Souza FA, Fernandez F, Séjalon-Delmas N, Declerck S (2005) Arbuscular mycorrhizal fungi exhibit distinct pattern of anastomoses formation and hyphal healing mechanism between different phylogenic groups. New Phytol 165:261-271

Engelmoer DJP, Kiers ET (2015) Host diversity affects the abundance of the extraradical arbuscular mycorrhizal network. New Phytol 205:1485-1491

Fiorilli V, Lanfranco L, Bonfante P (2013) The expression of GintPT, the phosphate transporter of Rhizophagus irregularis, depends on the symbiotic status and phosphate availability. Planta 237:1267-1277

Friese C, Allen MF (1991) The spread of VA mycorrhizal fungal hyphae in the soil: inoculum types and external hyphal architecture. Mycologia 83:409-418

Giovannetti M, Avio L (2002) Biotechnology of arbuscular mycorrhizas. In: Khachatourians GG, Arora DK (eds) Applied mycology and biotechnology, vol 2, Agriculture and food production. Elsevier, Amsterdam, NL, pp 275-310

Giovannetti M, Mosse B (1980) An evaluation of techniques to measure vesicular-arbuscular mycorrhizal infection on roots. New Phytol 84:489-500

Giovannetti M, Azzolini D, Citernesi AS (1999) Anastomosis and nuclear and protoplasmic exchange in arbuscular mycorrhizal fungi. Appl Environ Microbiol 65:5571-5575

Giovannetti M, Fortuna P, Citernesi AS, Morini S, Nuti MP (2001) The occurrence of anastomosis formation and nuclear exchange in intact arbuscular mycorrhizal networks. New Phytol 151:717-724

Giovannetti M, Sbrana C, Strani P, Agnolucci M, Rinaudo V, Avio L (2003) Genetic diversity of isolates of Glomus mosseae from different geographic areas detected by vegetative compatibility testing and biochemical and molecular analysis. Appl Environ Microbiol 69:616-624

Giovannetti M, Sbrana C, Avio L, Strani P (2004) Patterns of below-ground plant interconnections established by means of arbuscular mycorrhizal mycorrhizal networks. New Phytol 164:175-181

Giovannetti M, Avio L, Sbrana C (2015) Functional significance of anastomosis in arbuscular mycorrhizal networks. In: T.R. Horton (ed) Mycorrhizal networks. Springer, Dordrecht, pp 41-67

Glass L, Kaneko I (2003) Fatal attraction: nonself recognition and heterokaryon incompatibility in filamentous fungi. Eukaryotic Cell 2:1-8 
Gonzalez-Guerrero M, Azcon-Aguilar C, Mooney M, Valderas A, MacDiarmid CW, Eide DJ, Ferrol N (2005) Characterization of a Glomus intraradices gene encoding a putative $\mathrm{Zn}$ transporter of the cation diffusion facilitator family. Fungal Genet Biol 42:130-140

Helgason T, Daniell TJ, Husband R, Fitter AH, Young JPW (1998) Ploughing up the wood-wide web? Nature $394-431$

Krüger M, Krüger C, Walker C, Stockinger H, Schüßler A (2012) Phylogenetic reference data for systematics and phylotaxonomy of arbuscular mycorrhizal fungi from phylum to species level. New Phytol 193:970-984

Lopez-Pedrosa A, Gonzalez-Guerrero M, Valderas A, Azcon-Aguilar C, Ferrol N (2006) GintAMT1 encodes a functional high-affinity ammonium transporter that is expressed in the extraradical mycelium of Glomus intraradices. Fungal Genet Biol 43:102-110

Mikkelsen BL, Rosendahl S, Jakobsen I (2008) Underground resource allocation between individual networks of mycorrhizal fungi. New Phytol 180:890-898

Phillips JM, Hayman DJ (1970) Improved procedures for clearing roots and staining parasitic and vesiculararbuscular mycorrhizal fungi for rapid assessment of infection. Trans Brit Mycol Soc 55:158-161

Purin S, Morton JB (2011) In situ analysis of anastomosis in representative genera of arbuscular mycorrhizal fungi. Mycorrhiza 21:505-514

Purin S, Morton JB (2013) Anastomosis behaviour differs between asymbiotic and symbiotic hyphae of Rhizophagus clarus. Mycologia 12:589-602

Ravnskov S, Jakobsen I (1995) Functional compatibility in arbuscular mycorrhizas measured as hyphal P transport to the plant. New Phytol 129:611-618

Sbrana C, Fortuna P, Giovannetti M (2011) Plugging into the network: belowground connections between germlings and extraradical mycelium of arbuscular mycorrhizal fungi. Mycologia 103:307-316

Simard SW, Durrell DM (2004) Mycorrhizal networks: a review of their extent, function and importance. Can J Bot 82:1140-1165

Smith SE, Gianinazzi-Pearson V (1990) Phosphate uptake and arbuscular activity in mycorrhizal Allium cepa L.: effects of photon irradiance and phosphate nutrition. Aust J Plant Physiol 17:177-188

Smith SE, Smith FA, Jakobsen I (2004) Functional diversity in arbuscular mycorrhizal (AM) symbioses: the contribution of the mycorrhizal $\mathrm{P}$ uptake pathway is not correlated with mycorrhizal responses in growth or total P uptake. New Phytol 162:511-524

Voets L, de la Providencia IE, Declerck S (2006) Glomeraceae and Gigasporaceae differ in their ability to form mycelium networks. New Phytol 172:185-188 